THE ASTROPHYSICAL JOURNAL, 516:924-933, 1999 May 10

(C) 1999. The American Astronomical Society. All rights reserved. Printed in U.S.A.

\title{
DYNAMIC PLANAR MAGNETIC RECONNECTION SOLUTIONS FOR INCOMPRESSIBLE PLASMAS
}

\author{
I. J. D. CRAig ${ }^{1}$ and P. G. Watson ${ }^{1}$ \\ Received 1998 July 23; accepted 1998 December 15
}

\begin{abstract}
The planar magnetic reconnection problem for viscous, resistive plasmas is addressed. We show that solutions can be developed by superposing transient nonlinear disturbances onto quiescent "background" fields. The disturbance fields are unrestricted in form, but the spatial part of the background field must satisfy $\nabla^{2} K=-\lambda K$. This decomposition allows previous analytic reconnection solutions, based on one-dimensional disturbance fields of "plane wave" form, to be recovered as special cases. However, we point out that planar disturbance fields must be fully two-dimensional to avoid the pressure problem associated with analytic merging models, that is, to avoid unbounded current sheet pressures in the limit of small plasma resistivities. The details of the reconnection problem are then illustrated using cellular background field simulations in doubly periodic geometries. The flux pile-up rate is shown to saturate when the pressure of the current sheet exceeds the hydromagnetic pressure of the background field. Although the presaturation regime is well described by one-dimensional current sheet theory, the nonlinear postsaturation regime remains poorly understood. Preliminary evidence suggests that, although after saturation the early evolution of the field can be described by slow Sweet-Parker scalings, the first implosion no longer provides the bulk of the energy release.
\end{abstract}

Subject headings: MHD — plasmas — waves

\section{INTRODUCTION}

Magnetic reconnection can occur when topologically separate magnetic flux regions are driven together. Topological change is effected by cutting and rejoining field lines at localized sites determined by the null-points of the field (e.g., Parker 1979). It is suspected that the energy liberated in magnetic merging can be huge, perhaps sufficient to power the explosive release of the solar flare. The problem is that, for weakly resistive astrophysical plasmas, a collapse to extremely small length scales has to be involved if a physically significant reconnection rate is to be achieved. The difficulties of modeling a global nonlinear process, involving localized sites of energy release, has undoubtedly inhibited the development of plausible magnetic reconnection mechanisms.

The problem of incompressible merging in two space dimensions provides the simplest setting for magnetic reconnection. Even so, a plausible description valid at realistic plasma resistivities has proved elusive. Analytic solutions have long been available for the merging of straight field lines - that is, magnetic annihilation-but these involve no change in the field topology (Clark 1964; Sonnerup \& Priest 1975). As a result, reconnection theory has often tended to rely on a controversial mixture of quasilinear, semianalytic modeling and numerical simulations at unrealistically high plasma resistivities (see reviews by Forbes \& Priest 1987 and Biskamp 1994).

Recently, however, it has become clear that exact analytic solutions can be developed for the nonlinear reconnection problem. The essential step is to allow shearing motions, as well as compressive flows, to drive the reconnection (Craig $\&$ Henton 1995). Such solutions were initially constructed for planar incompressible merging, but they generalize very naturally to time-dependent, three-dimensional situations (Craig \& Fabling 1996, 1998; Watson \& Craig 1997a,

\footnotetext{
${ }^{1}$ Department of Mathematics, University of Waikato, Private Bag 3105, Hamilton, New Zealand; i.craig, pgwatson@math.waikato.ac.nz.
}

1997b; Craig \& McClymont 1997). The main limitation of these solutions is the "reduced dimensionality" of the intense current structures that produce the ohmic dissipation. These are current sheets in "fan reconnection" and tubular structures in "spine reconnection." Either way, there is at least one ignorable dimension along which the current uniformly extends.

A related difficulty is the "pressure problem" experienced by all analytic merging solutions (see Inverarity \& Priest 1996; McClymont \& Craig 1996). It is found that unbounded hydromagnetic pressures are required to sustain the dissipation rate in the limit of small plasma resistivities. Although on physical grounds the merging rate must always saturate at some level, the models developed so far do not saturate naturally; saturation has to be imposed externally by limiting the pressure in the reconnection region. This difficulty is an artifact of an analytic formulation that effectively negates the nonlinear feedback terms required for pressure saturation (see $\$ 3.7$ ).

The aim of the present paper is to construct more general reconnection solutions by extending the analytic formulation of the planar reconnection problem. The approach we adopt - which assumes that any field can be resolved into the superposition of a global, slowly evolving component and a transient, localized disturbance - is formally exact, time-dependent, and includes viscous as well as ohmic dissipation at the outset. This has the advantage of allowing all previous planar solutions, based on one-dimensional current sheets, to be recovered as special cases. The main drawback is that solutions involving two-dimensional current structures must be constructed numerically in general.

The problem is formulated in $\S 2$. In $\S 3$ we describe how previous analytic solutions can be interpreted within the present framework. In particular, we derive analytic scaling laws for the resistive dissipation and explain how the boundedness of the pressure implies a natural saturation of the reconnection rate. In $\S 4$ we present detailed 
reconnection solutions for the case of periodic geometries. We compare the analytic theory of $\S 3$ with computed scaling laws and describe the details of the merging rate saturation. Our conclusions are presented in $\S 5$.

\section{PLANAR RECONNECTION EQUATIONS}

\subsection{Introduction}

We assume that the plasma is governed by the incompressible, resistive MHD equations. We adopt nondimensional units in which fluid velocities are expressed in units of the Alfvén speed at some specified point in the reconnection region, which we take as the interior of the square $0 \leq|x|,|y| \leq 1$. The momentum and induction equations govern the evolution of the $\boldsymbol{v}$ and $\boldsymbol{B}$ fields. These have the general form

$$
\begin{gathered}
\frac{\partial v}{\partial t}+(\boldsymbol{v} \cdot \nabla) v=\boldsymbol{J} \times \boldsymbol{B}-\nabla p+v \nabla^{2} v \\
\frac{\partial \boldsymbol{B}}{\partial t}-\nabla \times(\boldsymbol{v} \times \boldsymbol{B})=\eta \nabla^{2} \boldsymbol{B}
\end{gathered}
$$

where $\boldsymbol{J}=\boldsymbol{\nabla} \times \boldsymbol{B}$ is the current density, $p$ is the plasma pressure, and we have normalized the density to unity. Here $v$ and $\eta$ are dimensionless constants that determine the level of viscous and resistive damping.

In the present case $z$ is an ignorable coordinate, and so we can use flux and stream function representations for the magnetic and velocity fields. With

$$
\boldsymbol{B}(x, y, t)=\nabla \psi \times \hat{z}, \quad \boldsymbol{v}(x, y, t)=\nabla \phi \times \hat{z},
$$

the constraints $\boldsymbol{\nabla} \cdot \boldsymbol{B}=\boldsymbol{\nabla} \cdot \boldsymbol{v}=0$ are automatically satisfied. The planar reconnection problem now reduces to coupled scalar equations for the potentials $\phi(x, y, t)$ and $\psi(x, y, t)$.

\subsection{Scalar Potential Equations}

We use the Poisson bracket notation,

$$
[\psi, \phi]=\psi_{x} \phi_{y}-\psi_{y} \phi_{x},
$$

with $\psi_{x}=\partial \psi / \partial x$ and so on, to write the momentum and induction equations in the form

$$
\begin{gathered}
\nabla^{2} \phi_{t}+\left[\nabla^{2} \phi, \phi\right]=\left[\nabla^{2} \psi, \psi\right]+v \nabla^{4} \phi, \\
\psi_{t}+[\psi, \phi]=\eta \nabla^{2} \psi .
\end{gathered}
$$

Note that we have eliminated the plasma pressure by working with the curled form of the momentum equation.

What is the signature of magnetic reconnection? A necessary condition for rapid topological change is the advection of flux across surfaces - magnetic separatrices - that delineate topologically distinct regions of the field. Since the bracket $[\psi, \phi]=(\boldsymbol{v} \times \boldsymbol{B}) \cdot \hat{z}$ represents the advection of the field by the flow, reconnection is signaled by a nonvanishing advection bracket on some separatrix of the field (as discussed by Watson \& Craig 1998).

\subsection{Global Energy Dissipation}

The sum of the resistive and viscous losses, that is,

$$
W_{\eta}+W_{v}=\int\left(\eta J^{2}+v \Omega^{2}\right) d V,
$$

where $\Omega=\nabla \times v=-\nabla^{2} \phi \hat{z}$ and $J=-\nabla^{2} \psi \hat{z}$ determines global energy losses of the fluid. In the present formulation the plasma resistivity $\eta$ is an inverse Lundquist number of order $10^{-12}$ in the solar corona. The ohmic dissipation rate $W_{\eta}$ is therefore negligible unless the plasma contains strong currents associated with near-singular regions of the magnetic field. In particular, if magnetic reconnection is to be significant as an energy conversion mechanism, current sheets must develop to maintain an appreciable ohmic dissipation rate.

Turning now to viscous dissipation and noting the ordering $\eta \ll v \ll 1$ (Spitzer 1967), it might be thought that the viscous losses $W_{v}$ should always dominate the global energy decay. But it should be remembered that only resistive reconnection can remove topologically bound magnetic energy from the system: viscosity, in common with all other nonresistive damping mechanisms, is ineffective in this regard. It follows that large current densities can be expected to accompany any disturbance that alters the field topology, independent of the level of the fluid viscosity.

\subsection{Quasi-steady and Transient Fields}

To develop solutions we break each scalar field into quasi-steady and transient components. As motivation, we note that in the usual model of antiparallel magnetic merging, a quiescent flow field is required to advect field lines towards the neutral point. The only small length scale that develops is associated with the steep magnetic field gradients of the current sheet. In more general reconnection solutions, which involve transient disturbances (nonlinear waves) superposed onto global magnetic fields, small length scales are present in the flow as well as the field. In all cases a global field is required.

In what follows we consider the decomposition

$$
\begin{aligned}
& \phi=\alpha(t) K(x, y)+f(x, y, t), \\
& \psi=\beta(t) K(x, y)+g(x, y, t),
\end{aligned}
$$

where $K$ represents the spatial part of a slowly evolving background field. We assume that $\alpha$ and $\beta$ change very slowly in comparison to the fields $f(x, y, t)$ and $g(x, y, t)$, which represent transient, rapidly evolving disturbances. The key point is that, for arbitrary amplitude disturbance fields, the momentum and inductions equations naturally split into quiescent and transient field components.

Note that the form we take for the background field corresponds to flow along the field lines when $f=g=0$ and $\beta$ is nonvanishing. Although, in principle, we can replace the background field in equation (9) by an arbitrary function of $K$ and still maintain this condition, we have not yet been able to exploit this freedom in constructing reconnection solutions.

\subsection{The Quasi-steady Field}

Consider the splitting of the induction equation (6). Assuming equations (8) and (9) we find that

$$
\begin{gathered}
g_{t}+[\beta K+g, \alpha K+f]=\eta \nabla^{2} g, \\
\dot{\beta} K=\eta \beta \nabla^{2} K .
\end{gathered}
$$

Equation (11) immediately implies that

$$
\dot{\beta}=-\eta \lambda \beta, \quad \nabla^{2} K=-\lambda K,
$$

where $\lambda$ is a separation constant.

These equations must be consistent with the momentum equation (5). The splitting of equation (5) gives the back- 
ground field equation

$$
\dot{\alpha} \nabla^{2} K+\left(\alpha^{2}-\beta^{2}\right)\left[\nabla^{2} K, K\right]=v \alpha \nabla^{4} K .
$$

The second term vanishes by equation (12), and so equation (13) naturally separates. Consistency requires that we again choose $\lambda$ as the separation constant. The only new constraint is $\dot{\alpha}=-v \lambda \alpha$, and so we conclude that

$$
\begin{gathered}
\alpha=\alpha_{0} \exp (-v \lambda t), \quad \beta=\beta_{0} \exp (-\eta \lambda t), \\
\nabla^{2} K=-\lambda K,
\end{gathered}
$$

determines the form of the background field. Note that, although our analysis is exact, we must choose $\lambda$ of order unity to maintain our interpretation of $K(x, y)$ as a global background field.

\subsection{The Disturbance Fields}

We return now to the equations for the disturbance fields. It is instructive to write these in a form that isolates the advection of the background flow. Using $\nabla^{2} K=-\lambda K$ from equation (14), the momentum equation gives

$$
\begin{aligned}
\nabla^{2} f_{t}+ & {\left[\left(\nabla^{2}+\lambda\right)\left(f-\frac{\beta}{\alpha} g\right), \alpha K\right] } \\
& =\left[\nabla^{2} g, g\right]-\left[\nabla^{2} f, f\right]+v \nabla^{4} f,
\end{aligned}
$$

while the induction equation (6) simplifies to

$$
g_{t}+\left[g-\frac{\beta}{\alpha} f, \alpha K\right]=\eta \nabla^{2} g+[f, g] .
$$

To pursue analytic solutions we must simplify some of the brackets. By setting brackets to zero we generally impose some form of conservation law or symmetry on the solution. One possibility is to explore one-dimensional disturbance fields for which brackets such as $\left[\nabla^{2} g, g\right]$ vanish identically. This construction in fact provides the basis of the analytic reconnection models discussed in $\S 3$.

\subsection{Boundary Conditions}

We have yet to discuss the symmetry or boundary conditions imposed on the merging problem. For incompressible merging the reconnection geometry of interest is either periodic or "open," in the sense that material can enter and leave the reconnection region. A combination of both conditions is also possible. For the moment we shall assume only that reconnection is driven by inflows through the physically equivalent surfaces $x= \pm 1$. In $\S 3$ we describe solutions that assume one-dimensional disturbance fields: these generally require open inflow and outflow boundaries; however, periodicity in one direction is also possible (as in $\S 3.8$ ). In $\S 4$ we concentrate on constructing twodimensional disturbance fields for doubly periodic solutions.

\section{THEORY OF ONE-DIMENSIONAL DISTURBANCE FIELDS}

\subsection{Introduction}

In this section we present a theoretical framework for incompressible merging based on the evolution of onedimensional disturbance fields. Our purpose is to derive simple analytic scaling laws for the energy dissipation, which can be used for comparison with more general merging problems, in particular, those associated with periodic reconnection geometries. We also show how an unphysical pressure scaling in limit $\eta \rightarrow 0$ is a consequence of assuming one-dimensional disturbance fields.

\subsection{Form of the Background Field}

The formalism of $\S 2$ makes it very easy to recover previous reconnection solutions. These are based on "plane wave" forms

$$
f=f(x, t), \quad g=g(x, t),
$$

which reduce many of the brackets to zero,

$$
[f, g]=\left[\nabla^{2} f, f\right]=\left[\nabla^{2} g, g\right]=0 .
$$

We note, for example, that the nonlinear "forcing terms" on the right-hand side of the momentum equation (15) automatically vanish. Physically, this implies that magnetic field disturbances can build up continually without feedingback on the flow, a problem to which we return in $\$ 3.7$.

The induction equation (16) can only be consistent with plane wave forms if $K(x, y)$ is a linear function of $y$. The possible solutions of equation (14) are therefore

$$
K=x y, \quad K=\sin (\kappa x) y, \quad K=\sinh (\kappa x) y,
$$

where $\kappa=|\lambda|^{1 / 2}$ is a constant of order unity. The first member of equation (19) provides the only timeindependent background field: $\alpha=\alpha_{0}, \beta=\beta_{0}$ from equation (14). This provides the basis for the fast, steady-state reconnection solution of Craig \& Henton (1995), which we discuss briefly in $\S 3.4$. Also of particular interest is the sinusoidal model $K=\sin (\kappa x) y$, which gives the possibility of periodic inflows into an assemblage of neutral points (see $\S 3.8)$.

\subsection{Disturbance Potentials for $K=x y$}

The harmonic field $K=x y$ allows the simplest reconnection solutions. On integrating the momentum equation (15) twice, the equations for the disturbance potentials reduce to

$$
\begin{gathered}
f_{t}+\alpha x f_{x}=2 \alpha f-2 \beta g+\beta x g_{x}+v f_{x x}, \\
g_{t}+\alpha x g_{x}=\beta x f_{x}+\eta g_{x x} .
\end{gathered}
$$

An important property of this system is that, for an inviscid plasma or for a plasma in which $v$ scales linearly with resistivity, the explicit dependence on $\eta$ can be removed by letting $x \rightarrow \eta^{1 / 2} x$. In such cases a universal small length scale $x \sim \eta^{1 / 2}$ can be associated with the merging problem.

Equations (20) and (21) must generally be solved numerically as a coupled system. Analytic progress can be made, however, if we exploit the fact that global initial disturbances must localize sufficiently before rapid dissipation can set in (see § 3.5). An alternative approach is to seek reconnection solutions by specializing to the case of a steady-state, inviscid plasma.

\subsection{Steady-State Reconnection: $K=x y$}

In steady-state, the inviscid momentum equation (20) is satisfied by taking $f=\beta g / \alpha$. We assume $\beta \geq 0$ for definiteness and take $\alpha<0$ corresponding to inflow towards the plane $x=0$.

The induction equation (21) governs the form of $g(x)$ :

$$
E+\alpha^{-} x g^{\prime}=\eta g^{\prime \prime}, \quad \alpha^{-} \equiv \frac{\left(\alpha^{2}-\beta^{2}\right)}{\alpha},
$$

where $E \equiv g_{t}$ is a constant that determines the rate of flux annihilation. Equation (22) has the formal hypergeometric 
function solution

$$
g(x)=\frac{E}{\eta} \frac{x^{2}}{2}{ }_{1} F_{2}\left(1, \frac{3}{2}, 2, \frac{\alpha^{-}}{2 \eta} x^{2}\right),
$$

from which the physical properties of the magnetic merging can be deduced.

These equations describe the fast reconnection solution of Craig \& Henton (1995). The solution evidently requires an open geometry, in which inflow through the surfaces $x= \pm 1(\alpha<0)$ is matched by outflow through $y= \pm 1$. For well-behaved solutions we also require $\alpha^{2}>\beta^{2}$ (see $\S 3.5)$. Under these conditions the flow exhibits a narrow shearing layer aligned to the current sheet of width $\Delta x \sim \eta^{1 / 2}$ in which $J \sim \eta^{-1}$. The case $\beta=0$ provides an exception: all disturbance flows vanish, and the annihilation solution of Sonnerup \& Priest (1975) is recovered. But in all cases the ohmic dissipation rate is superfast, $W_{\eta} \sim$ $\eta J^{2} \Delta x \sim \eta^{-1 / 2}$. This rate is maintained against reductions in $\eta$ by the increasing intensity of the "flux pile-up" field at the onset of the current layer.

It is important to emphasize that the main features of the solution - in particular, the scaling laws for the ohmic dissipation - remain robust to the inclusion of fluid viscosity (Fabling \& Craig 1996). We now show that similar scaling laws can be predicted on the basis of a completely dynamic treatment.

\subsection{Time-dependent Disturbance Fields: $K=x y$}

The disturbances in equations (20) and (21) are plane waves that, under the conditions of fast reconnection, localize and grow as they approach the neutral point $x=0$. We can derive scaling laws for the ohmic dissipation if we assume an initial advection phase in which global disturbances localize without appreciable energy losses, an assumption justified by the smallness of the resistivity.

For the moment we work with the magnetic and velocity fields rather than with the potentials. Writing

$$
V(x, t)=-f_{x}, \quad Y(x, t)=-g_{x},
$$

and introducing the comoving frame

$$
\tau=t, \quad \xi=x \exp (-\alpha t),
$$

recovers the inherent symmetry in the velocity and magnetic fields:

$$
\begin{aligned}
& V_{\tau}=\beta \xi Y_{\xi}+\alpha V-\beta Y+v e^{-2 \alpha \tau} V_{\xi \xi}, \\
& Y_{\tau}=\beta \xi V_{\xi}-\alpha Y+\beta V+\eta e^{-2 \alpha \tau} Y_{\xi \xi} .
\end{aligned}
$$

For the initial advection phase we set $v=\eta=0$ and eliminate $V$ from the system. The result is a Kline-Gordon equation for the field:

$$
Y_{\tau \tau}=\beta^{2} Y_{\chi \chi}+\left(\alpha^{2}-\beta^{2}\right) Y, \quad \chi=\ln \xi .
$$

The possibility of growth in the solution follows from the final term in equation (28); a necessary condition is clearly $\alpha^{2}-\beta^{2}>0$. However, the second term shows that wave modes are present for $\beta \neq 0$ governed by the characteristics $\chi \pm \beta \tau$. These modes arise from tension (curvature) in the field lines and are oscillatory in character: they reflect the elastic properties of the magnetic medium and are absent in the purely compressional annihilation mode $\beta=0$. In real space the characteristics are given by

$$
C^{+}=x e^{-(\alpha-\beta) t}, \quad C^{-}=x e^{-(\alpha+\beta) t},
$$

and so with $\alpha<0$ all disturbances are inward propagating, provided that $|\alpha|>\beta$. These conditions are necessary for growth in the field.

\subsection{Fast and Slow Reconnection Solutions: $K=x y$}

How are the characteristics related to the formation of current sheets in the fluid? Let us first dispense with the case of slow reconnection $\alpha^{2}<\beta^{2}$. Although global disturbances can rapidly localize to the $\eta^{1 / 2}$ length scale (on the $C^{+}$ characteristic with $\alpha<0$ ), the absence of growth in the field implies that only relatively weak current sheets can form. Such sheets obey the slow Sweet-Parker scalings $Y \sim \eta^{0}$, $J \sim \eta^{-1 / 2}, W_{\eta} \sim \eta^{1 / 2}$, as discussed in $\S 3.8$.

More interesting is the case of fast reconnection. A detailed analysis of the (three-dimensional) problem has been given by Craig \& Fabling (1998), and we shall provide only a summary discussion here. The key point is that, for global initial disturbances, the advection phase has two timescales determined by the rates

$$
\alpha^{ \pm}=\frac{\alpha^{2} \pm \beta^{2}}{\alpha} .
$$

These rates are negative under the growth/localization conditions $\alpha<0, \alpha^{2}>\beta^{2}$. The rapid $\alpha^{+}$timescale is associated with "equalizing" the magnetic and velocity fields (at the level $V \simeq \beta Y / \alpha$ ) outside the current layer. Large currents are built up on a much slower timescale, at least for $\eta$ sufficiently small. The rapidly localizing equalization wave acts as a precursor for the slow field build-up, which takes the form

$$
Y(x, t) \simeq Y_{0}\left(\xi^{-}\right) e^{-\alpha^{-} t}, \quad \xi^{-}=x e^{-\alpha^{-} t},
$$

where $Y_{0}$ is the initial field profile.

The growth of the field is eventually arrested by resistive diffusion (see also Zweibel 1998). We can estimate a current localization time by assuming that $\eta Y_{x x}$ eventually becomes comparable to $Y_{t}$ :

$$
T \simeq \frac{1}{2\left|\alpha^{-}\right|} \ln \frac{\left|\alpha^{-}\right|}{\eta} .
$$

By this time a current layer of width $\Delta x \sim \xi^{-} e^{\alpha^{-} T} \sim \eta^{1 / 2}$ has been achieved. Accordingly, we determine the "current maximum " scalings

$$
Y \sim \eta^{-1 / 2}, \quad J \sim \eta^{-1}, \quad W_{\eta} \simeq \eta J^{2} \Delta x \sim \eta^{-1 / 2} .
$$

These rates are consistent with those predicted by the steady-state, fast reconnection model of $\S 3.5$. Note that, although the equalization phase is absent in the case of magnetic annihilation, equations (32) and (33) can still be applied (with $\beta=0$ ).

\subsection{The Pressure Problem for Fast Reconnection Solutions:

$$
K=x y
$$

Using the primitive momentum equation (1), we recover the plasma pressure in the reconnection region:

$$
p(x, y, t)=p_{0}(t)-\frac{1}{2}\left[\alpha^{2}\left(x^{2}+y^{2}\right)+Y^{2}\right]-\beta y Y .
$$

The fact that $Y^{2}$ scales as $\eta^{-1}$ for fast reconnection implies that the pressure must increase to unphysical levels as $\eta \rightarrow 0$. This is the pressure problem mentioned in $\S 1$. The physical implication is that the disturbance field amplitudes 
$\phi$

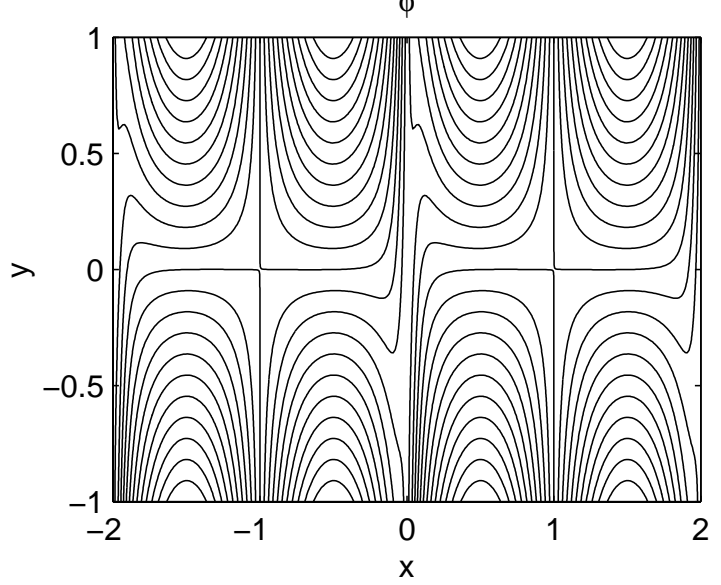

$\psi$

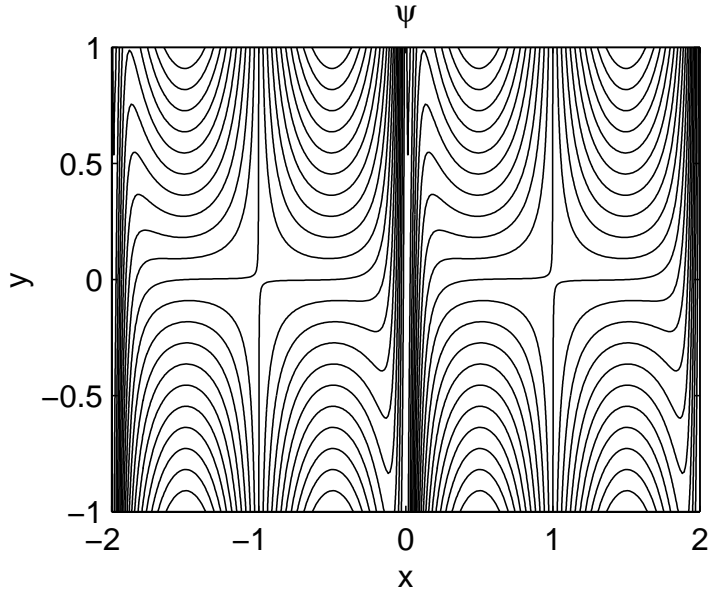

FIG. 1.-Typical fast reconnection solution for a singly periodic one-dimensional disturbance field. The solution is shown at the time of maximum current for the time-dependent background field model with $K=\sin (\kappa x) y$.

cannot be arbitrary; rather, they must be limited to levels that ensure plausible pressures at realistic plasma resistivities. Obviously, it makes little physical sense to allow the pressure in the sheet to exceed the "external" hydromagnetic pressures that drive the inflows. The fact that this limit must be imposed externally - by restricting the field amplitudes on the inflow boundaries - can be viewed as a defect of the present analytic treatment that arises as an artifact of taking strictly one-dimensional disturbance fields.

\subsection{Time-dependent Background Fields: $K=\sin (\kappa x) y$}

We now consider singly periodic, one-dimensional disturbance fields associated with the potentials

$\phi=\alpha(t) y \sin (\kappa x)+f(x, t), \quad \psi=\beta(t) y \sin (\kappa x)+g(x, t)$,

where $\alpha$ and $\beta$ satisfy equation (14). These forms allow the possibility of modeling a periodic assemblage of neutral

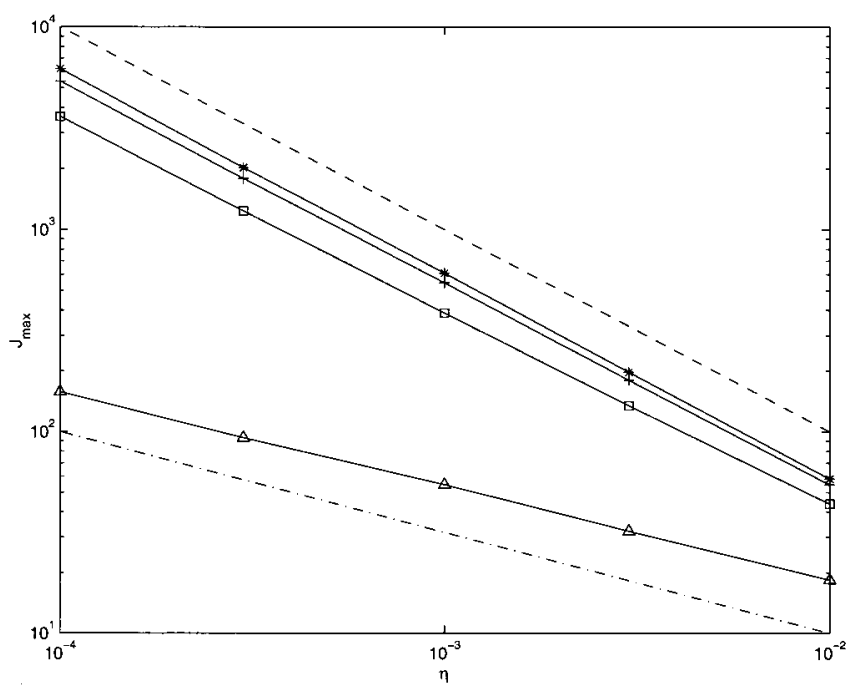

FIG. $2 a$ points, aligned along the $x$-axis: specifically, the choice $\kappa=\pi$ corresponds to inflow planes at $x=0, x= \pm 2$, $x= \pm 4 \ldots$

A typical fast solution, determined by a numerical treatment of equation (35), is shown in Figure 1. These profiles $\left(\alpha_{0}=-1, \beta_{0}=0.5, \eta=0.003, v=0\right)$ were obtained at the time of maximum current and derive from the initial conditions $f=0, g(x, 0)=\sin (\pi x)$.

Although the previous theory cannot strictly be applied to sinusoidal inflows, we expect the physical arguments to remain valid. In particular, the scalings of equation (33) might be expected to apply to any inflow profile that approximates stagnation point flow near the neutral point. Figure $2 a$ confirms that, for $\beta_{0}=0,0.25,0.5$, the computed maximum current follows very closely the fast $J \sim \eta^{-1}$ scaling of equation (31). An exception is provided by the case $\beta_{0}=1.1$, which has a slow $J \sim \eta^{-1 / 2}$ scaling, as expected by violation of the growth condition $\alpha^{2} \geq \beta^{2}$. Similarly, the computed localization time of Figure $2 b$ (identified as the time of current maximum) is in good accordance with the expected $\ln \eta$ trend of equation (32).

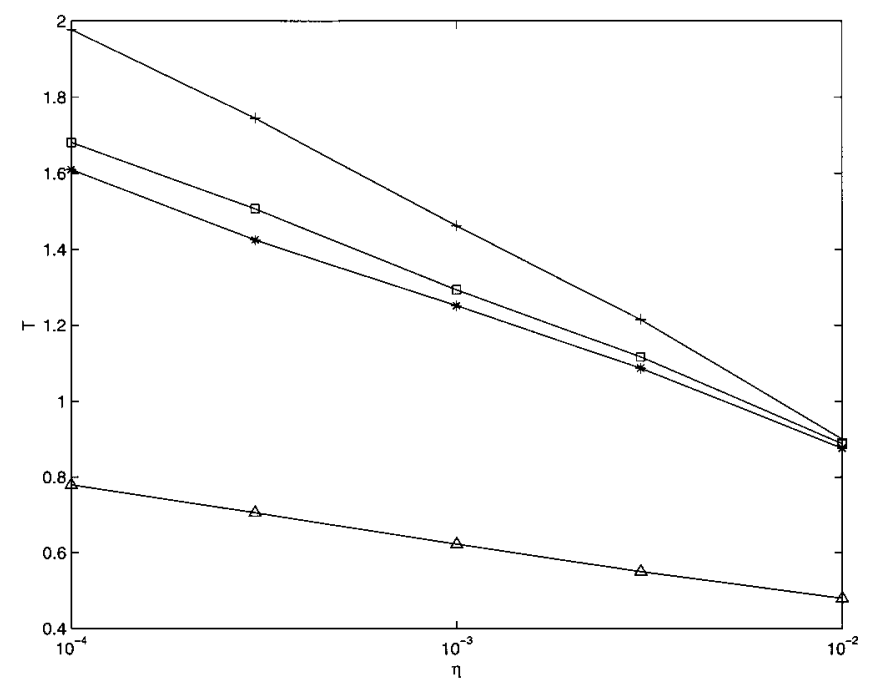

FIG. $2 b$

FIG. 2. - (a) Plot of the maximum current vs. resistivity for the time-dependent background field model $K=\sin (\kappa x) y$ for $\beta_{0}=0($ asterisks $), \beta_{0}=0.25$ (crosses), $\beta_{0}=0.5$ (squares), and $\beta_{0}=1.1$ (triangles). The upper dashed line represents the fast theoretical scaling $J \sim \eta^{-1}$, and the lower dashed line refers to the slow $J \sim \eta^{-1 / 2}$ scaling. (b) Localization time (the time to current maximum) vs. resistivity for the same runs described in $(a)$. 
This time is considerably reduced for the case $\beta_{0}=1.1$, since the current maximum now occurs on the timescale of the fast $C^{+}$characteristic: there is no slow build-up of the field as in the fast reconnection solutions. Of course, a strict identification with $T$ in equation (32) makes little sense, since $\beta$ is time-dependent in the present calculation.

The present computations also illustrate the pressure problem of $\S 3.7$. Since plane wave disturbance fields cannot feed back on the background flow-the brackets in equation (18) vanish - the scalings of Figure 2 can be extrapolated down to arbitrarily small values of $\eta$. It follows that the pressure in the sheet must rise to unphysical levels as $\eta \rightarrow 0$.

Finally, we mention a further problem associated with one-dimensional sheets sustained by sinusoidal inflows. If the pressure is regarded as unconstrained, the problem admits solutions in which the disturbance fields grow exponentially with time at a rate independent of the plasma resistivity! This unphysical behavior is the result of energy being bled out of the infinite background fields. Twodimensional disturbance fields eliminate this problem.

\section{TWO-DIMENSIONAL SOLUTIONS IN DOUBLY PERIODIC GEOMETRIES}

\subsection{Introduction}

We now turn to developing reconnection solutions in doubly periodic geometries. As a background field we take

$$
\begin{gathered}
\alpha=-\exp \left(-2 v \pi^{2} t\right), \quad \beta=\beta_{0} \exp \left(-2 \eta \pi^{2} t\right), \\
K(x, y)=\frac{1}{\pi} \sin (\pi x) \sin (\pi y),
\end{gathered}
$$

corresponding to the wavenumber $\kappa=\pi$. The initial form of the magnetic disturbance is one-dimensional

$$
f(x, y, 0)=0, \quad g(x, y, 0)=\frac{g_{0}}{\pi} \cos (\pi x),
$$

but a two-dimensional solution eventually develops. Note that the background field $K$ has period 2 in both $x$ and $y$, and therefore the disturbance fields must also be periodic over this domain. The frequency of the disturbance field can in fact be any integer multiple of this fundamental frequency.

We construct solutions by means of a centered finite difference scheme for the potentials of equations (5) and (6). In practice the momentum equation (5) updates the auxiliary vorticity variable $\Omega=-\nabla^{2} \phi$ : the velocity potential $\phi$ is then recovered by inverting the fast Fourier transform of $\Omega$. We use a two-step explicit code whose stability, for small resistivities and viscosities, is determined by the CFL condition.

\subsection{The Role of $\beta$ in Merging Solutions}

Figure 3 shows contours of the stream and flux function at the time of current maximum for a typical run $\left(\eta=v=0.001, \alpha_{0}=-1, \beta_{0}=0.5, g_{0}=0.1\right)$. These results confirm that the magnetic and velocity fields have the neutral point structure anticipated by the Craig-Henton solution (§3.4). As expected, the current is concentrated into quasi-one-dimensional sheets whose length extends throughout most of the convection cell. The vorticity also has an equally rich structure. Evidence of shearing in the flow profile is apparent but relatively minor. The shearing gradually disappears as $\beta_{0} \rightarrow 0$.

The numerical results suggest that $\beta$ loses some of its significance for two-dimensional disturbance fields. Recall that, in the analytic solutions of $\S 3$, a finite $\beta$ was required to obtain true $X$-point merging, that is, to obtain reconnection rather than annihilation solutions. In the present example all current sheets are of finite length, and in the absence of shearing $(\beta=0)$ all separatrices are physically identical. This accords with the classical reconnection picture in which both diffusion and advection are required to transfer flux across the separatrices (Watson, Priest, \& Craig 1998). The role of $\beta$ therefore is to transfer energy from the magnetic field to the velocity field by inducing a shearing component in the flow. Note that (e.g., in Fig. 5c) the presaturation velocity amplitudes increase with $\beta$. It follows that $\beta$ influences the strength of the magnetic slingshot that evacuates plasma from the vicinity of the neutral point.

\subsection{Nonlinear Saturation}

Aside from the phenomenology of magnetic merging, we are interested in the scalings of the current sheets and the possibility of stalling at sufficiently low values of the plasma resistivity. To this end it is convenient to choose $g_{0}$ small enough to ensure that disturbance fields of order unity are achieved for computationally accessible resistivities, say $\eta=O\left(10^{-3}\right)$. In Figure 3, the disturbance fields are beginning to interact with the background field, but the effect is not yet sufficient to induce stalling; further reductions in $\eta$ are required.

We expect stalling to occur when the magnetic pressure in the current sheet becomes comparable to the $O(1)$ hydromagnetic pressure associated with the background field. Figure 4 shows the effect that varying the initial field amplitude $g_{0}$ has on the ohmic dissipation rate for a typical set of simulations $\left(\beta_{0}=0\right)$. Although the standard run $\left(g_{0}=0.3\right)$ shows a fall-off in the dissipation rate for $\eta<10^{-3}$, the decline is postponed to lower resistivities for smaller amplitude disturbances $\left(g_{0}=0.1,0.03,0.01\right.$, respectively). Equally noteworthy, the fast scaling for the low-amplitude perturbations $g_{0}=0.01,0.03$ closely approximates the analytic prediction of $\S 3.6$, namely, $W_{\eta} \sim \eta^{-1 / 2}$, represented by the lower dashed line in the diagram. This suggests that the plane wave merging theory provides a useful model for fast reconnection, at least in the presaturation regime.

What happens when field saturation occurs? By assuming that the field saturates at a level independent of resistivity and taking an $\eta^{1 / 2}$ length scale for the quasi-onedimensional sheet that develops, we obtain the following scalings:

$$
Y \sim \eta^{0}, \quad J \sim \eta^{-1 / 2}, \quad W_{\eta} \sim \eta^{1 / 2} .
$$

Note we have used the notation of $\S 3$ and used $Y$ to represent the magnitude of the $y$-component of the magnetic field. It is instructive to compare these scalings with the scalings of the presaturation regime, given by equation (33). The new slow $\eta^{1 / 2}$ scaling for $W_{\eta}$, essentially an instantaneous Sweet-Parker relation, is shown by the upper dashed line in Figure 4.

Figure 5 shows the effect of the field saturation for three different values of $\beta_{0}$. The results were obtained for a fixed initial disturbance amplitude $g_{0}=0.3$, and they refer to the first implosion only. Figure $5 a$ shows the turnover from a 

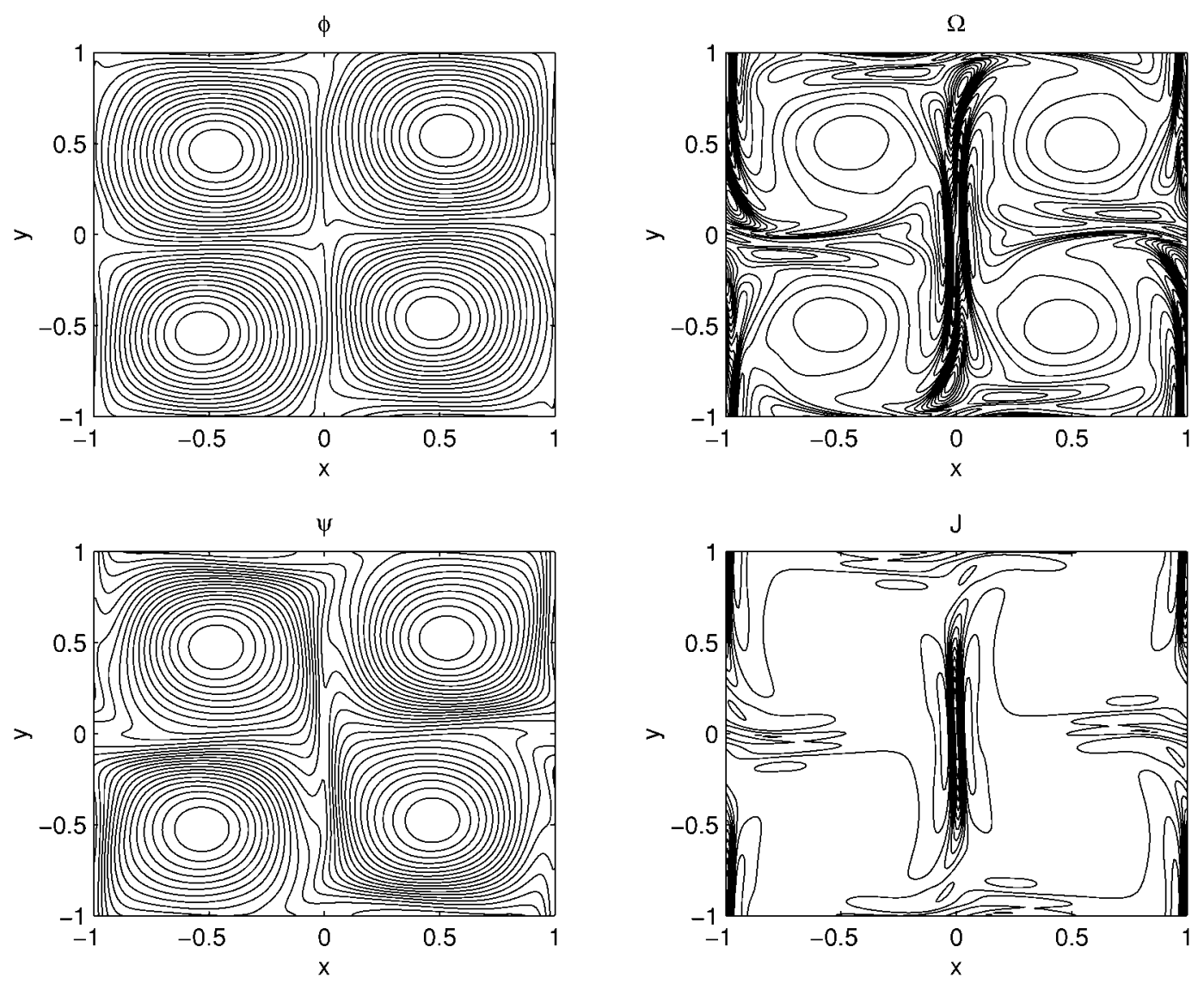

FIG. $3 a$

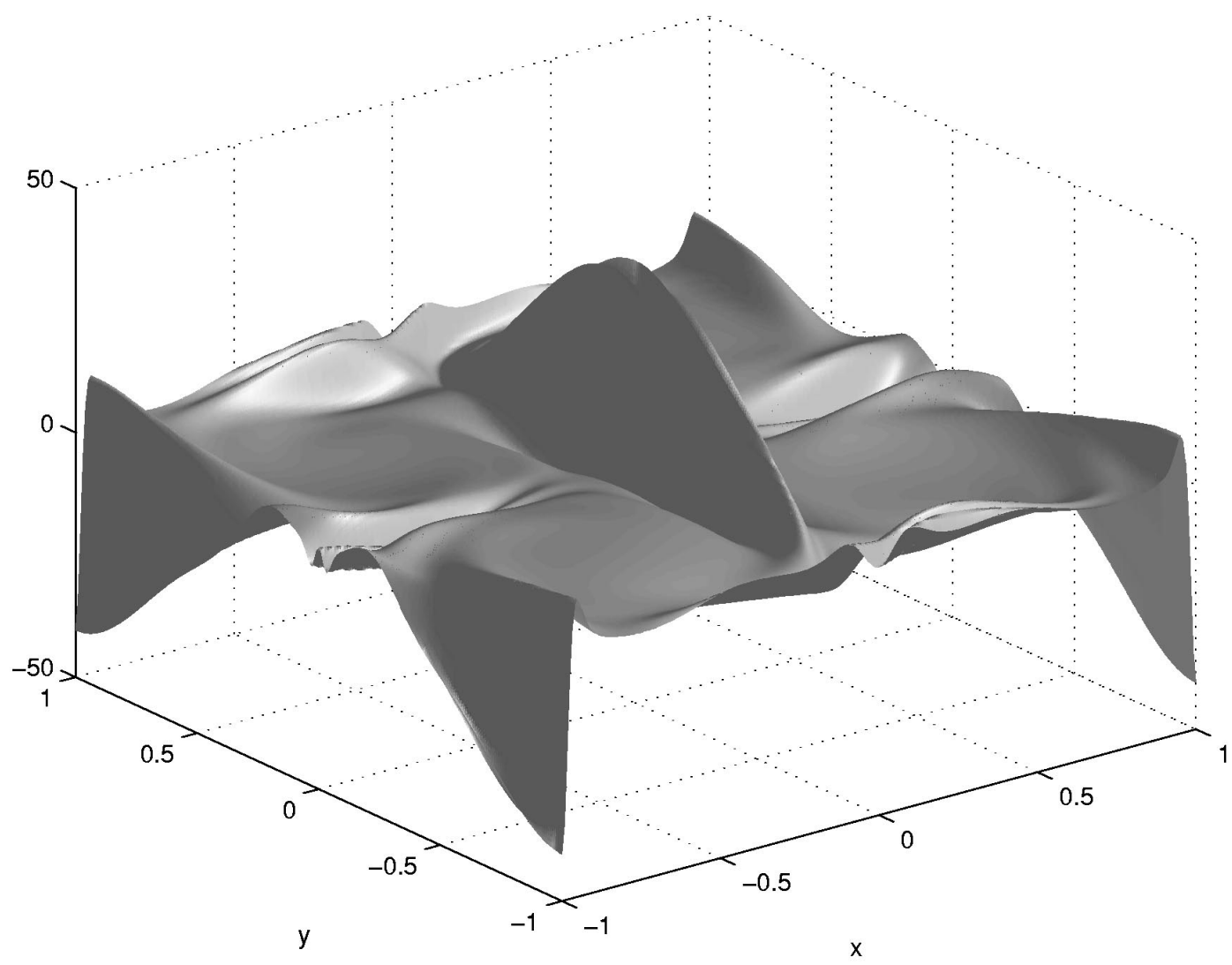

FIG. $3 b$

Fig. 3.-(a) Contour plots of the stream and flux functions and current and vorticity for a fast reconnection solution in doubly periodic geometry. This solutions has the background field $K=\sin (\pi x) \sin (\pi y) / \pi$ and the contours are drawn at the time of maximum current. $(b)$ Surface plot of the current. 


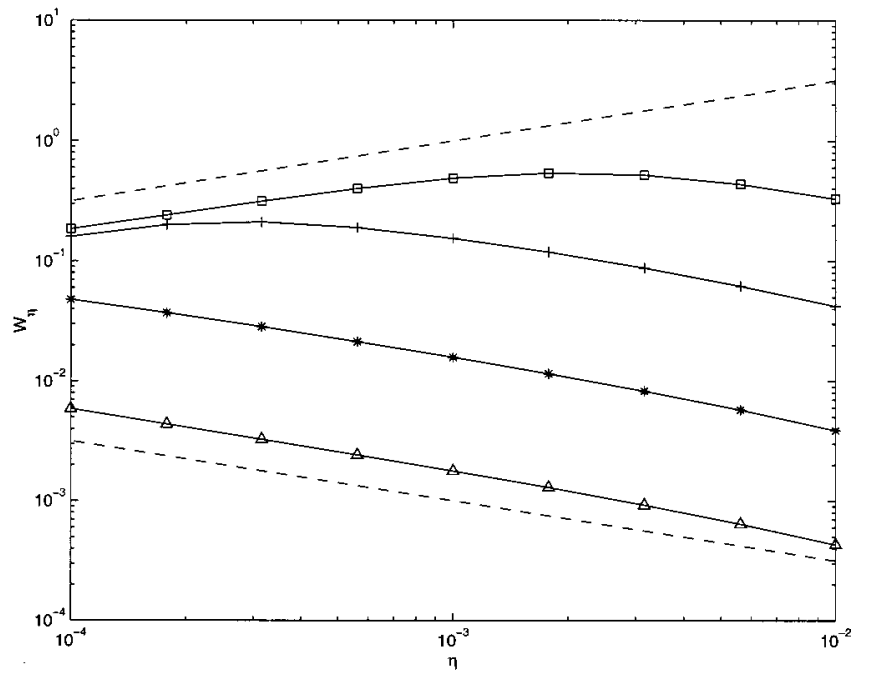

FIG. 4.-Plot of ohmic dissipation rate vs. resistivity with $\beta_{0}=0$ for four different values of the initial disturbance amplitude: $g_{0}=0.3$ (squares), $g_{0}=0.1$ (crosses), $g_{0}=0.03$ (asterisks), and $g_{0}=0.01$ (triangles).

fast to a slow ohmic dissipation rate, whereas Figure $5 b$ shows the change in the scaling for the peak current that occurs at the end of the first implosion. Figure $5 c$ shows a plot of the maximum field (solid lines) and velocities (dashed-dotted lines) at the time of peak current. The peak velocities are noticeably smaller than the peak magnetic fields in the fast reconnection regime, $\eta>10^{-3}$, especially for $\beta_{0}=0$. In fact there is quite a good agreement with the relation obtained from the steady-state analysis $f=\beta g / \alpha$ (see Craig \& Henton 1995). Once stalling has occurred, however, the disturbance speed becomes quite significant, certainly comparable to the speed of the background flow. Plots of the flow field for these saturated solutions confirm that the background field structure is strongly modified by the effect of back-pressures in the sheet.

\subsection{Beyond the First Implosion}

Unfortunately, the discussion on saturation given above and the straightforward modification of the onedimensional theory that goes with it are restricted to the first implosion. What happens after the first implosion is far more complicated. In the presaturation regime the peak current associated with the first implosion is always the largest current attained throughout the simulation. Once solutions enter the saturation regime this is no longer necessarily the case. Figures $6 a-6 c$ show plots of the maximum current over the computational mesh versus time. Figure $6 a$ shows the typical behavior of the maximum current for a presaturation solution. The first peak is the dominant one, and it is followed by a regular procession of subsequently weaker peaks. Figure $6 b$ shows a solution just at the onset of saturation. The first peak is no longer the dominant one; however, the regular pattern of the later peaks is maintained. Figure $6 c$ shows a solution well within the saturation regime. The later peaks have become decidedly more chaotic, and some of them are significantly larger than the first peak.

As yet we have no theory to explain the behavior past the first implosion. In the presaturation regime this does not present much of a problem, since the first implosion is the most significant phase of the reconnection process. Once

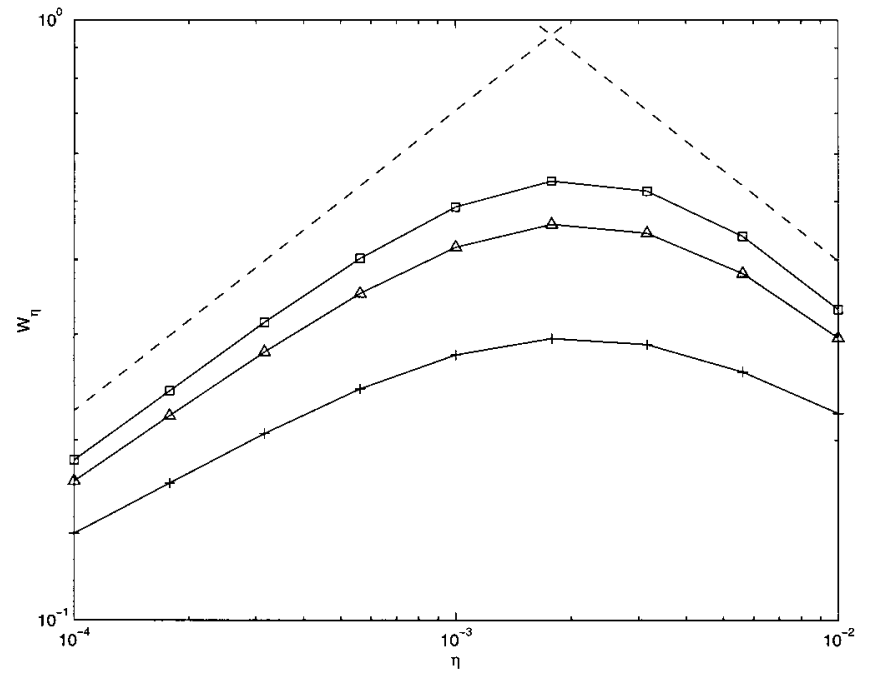

FIG. $5 a$

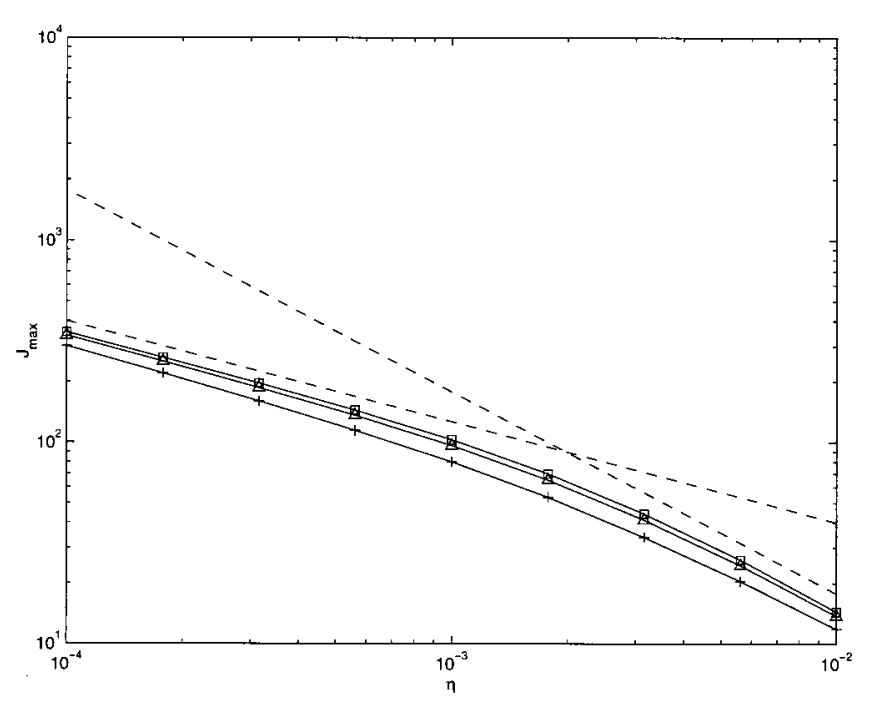

FIG. $5 b$

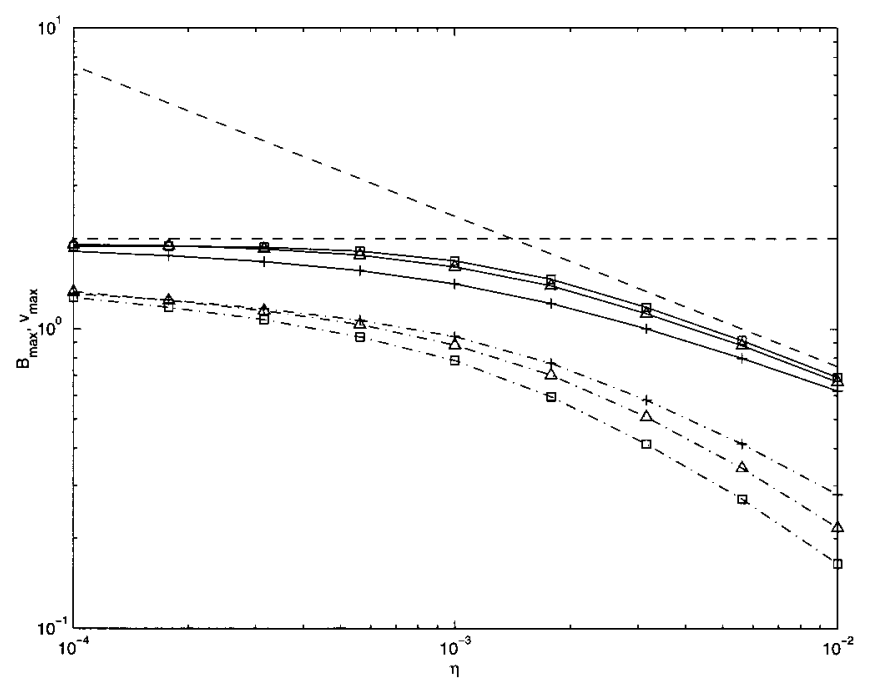

FIG. $5 c$

FIG. 5. - (a) Ohmic dissipation rate vs. resistivity for fixed amplitude initial disturbances, $g_{0}=0.3: \beta_{0}=0$ (squares), $\beta_{0}=0.25$ (triangles), and $\beta_{0}=0.5$ (crosses). (b) Maximum current vs. resistivity. (c) Maximum velocity and magnetic field vs. resistivity. In all cases the two dashed lines refer to the fast and slow theoretical scalings. 
(a)

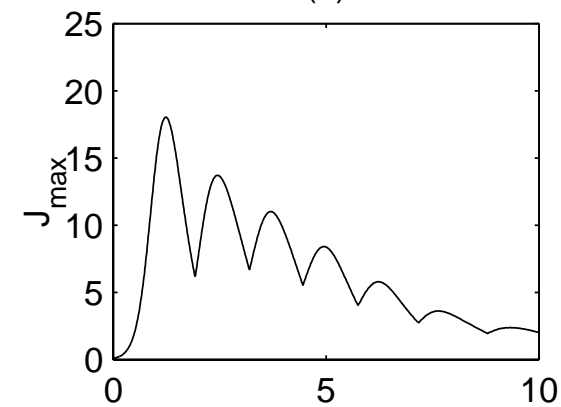

(b)

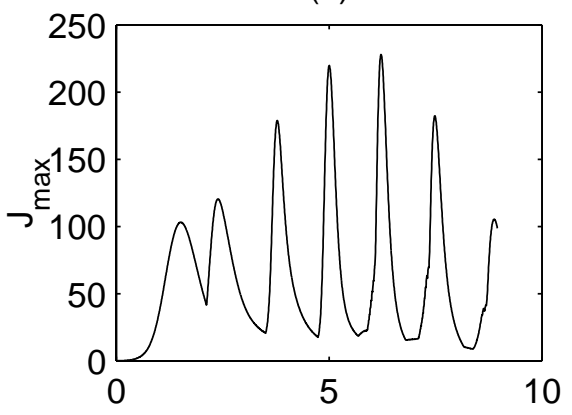

(c)

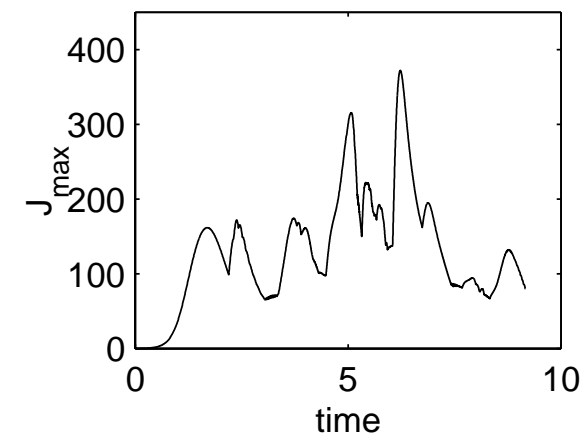

FIG. 6.-Plots of maximum current vs. time for $(a)$ a presaturation solution $\left(\eta=v=0.001, \beta_{0}=0\right.$, and $\left.g_{0}=0.03\right),(b)$ a solution at the onset of saturation $\left(\eta=v=0.000178, \beta_{0}=0\right.$, and $\left.g_{0}=0.03\right)$, and (c) a postsaturation solution $\left(\eta=v=0.0001, \beta_{0}=0.25\right.$, and $\left.g_{0}=0.03\right)$.

the solutions saturate, however, the nonlinear nature of the problem readily becomes apparent. A much stronger coupling between the disturbance fields and the background field develops, which rapidly destroys the quasi-onedimensional form of the problem. To our knowledge the phenomena of this interaction, and ensuing modifications of the dissipation rate, have not yet been extensively explored in literature (see brief discussions in Biskamp 1994; Craig, Henton, \& Rickard 1993; McClymont \& Craig 1996).

\subsection{Summary}

It is clear that two-dimensional disturbance fields offer a far more complete view of reconnection than the plane wave fields required by analytic theory of $\S 3$. In the first place we have seen that current sheet lengths are now finite and that reconnection occurs even for purely compressional merging with $\beta=0$, in contrast to the one-dimensional sheet theory.

However, in the presaturation regime, current sheets remain nearly one-dimensional, even in the cellular geometry. That is why the "superfast" analytic scaling laws hold to a good approximation, at least before saturation sets in. In agreement with previous arguments (Watson \&
Craig 1997a, 1997b; Craig, Fabling, \& Watson 1997), our examples show that saturation occurs when the disturbance field amplitudes become comparable to the amplitude of the background fields that drive the flow.

We have not yet addressed the question of what happens after the first implosion when the field amplitude saturates. Biskamp (1994) suggests that the reconnection rate reverts to Sweet-Parker, and this certainly seems to hold true for the first implosion. However, McClymont \& Craig (1996), using a compressible code in a closed $X$-point geometry, show that following the initial implosion, successive bounces can enhance the dissipation rate. Preliminary investigations using the present code also suggest that a kind of MHD pumping can lead to successively increasing current maxima in the saturation regime (see Fig. 6). To what extent the dissipation rate is enhanced by this behavior is unclear at present.

\section{CONCLUSIONS}

The planar reconnection problem for a resistive, viscous plasma has been discussed. We have shown that solutions can be constructed by superposing localized, transient disturbances onto large-scale, quiescent background fields. The background field must satisfy $\nabla^{2} K=-\lambda K$ to qualify as an appropriate global field (see $\S 2.3$ ). The disturbance fields are unrestricted in form; hence previous analytic reconnection solutions based on "plane wave" disturbances are easily recovered as special cases.

Analytic merging solutions were discussed in $\S 3$. Although reconnection solutions involving shearing flows can be developed, the assumption of plane wave disturbances, made for analytic tractability, restricts all current sheets to strict one-dimensionality. This effectively precludes the nonlinear "feed-back" between the disturbance fields and the background flow: formally the disturbance fields can have unrestricted amplitudes. The pressure problem - that is, the continual increase in the pressure in the reconnection region as $\eta \rightarrow 0$-is really an artifact of this effect. As a result, realistic merging rates can be deduced only by limiting, retrospectively, the hydromagnetic pressure of the current sheet to that of the background field. This fix is not required when the assumption of onedimensionality is dropped: the sheet pressure saturates naturally at a level depending on the plasma resistivity, and the disturbance field amplitudes.

In $\S 4$ we considered reconnection driven by a cellular, periodic background field, namely, $K \sim \sin (\pi x) \sin (\pi y)$. In the presaturation regime, quasi-one-dimensional current sheets were observed with the "superfast" scalings,

$$
\Delta x \sim \eta^{1 / 2}, \quad J \sim \eta^{-1}, \quad W_{\eta} \sim \eta^{-1 / 2} .
$$

This behavior accords with analytic models based on onedimensional disturbances in an open flow geometry (see $\S 3.5)$ and can lead to the energy dissipation characteristics of a modest flare (Craig et al. 1997). How these relationships are modified in the post saturation stage is largely unknown, but there is some evidence of significant dissipation even when the field amplitude is saturated. What can be said is that, although the saturated dissipation rate on the first implosion reverts to a slow Sweet-Parker scaling, the first bounce no longer appears to release the bulk of the free energy.

But even in the absence of saturation, we cannot expect scaling law (39) to hold universally: Watson \& Craig 1997b 
have already given an analytic example of multiple scalings when two neutral points are present. More specifically, in simulations of the coalescence instability (Biskamp \& Welter 1980; Rickard \& Craig 1993), the width of the sheet, prior to saturation, scales not as $\eta^{1 / 2}$ but as $\Delta x \sim \eta^{2 / 3}$. This scaling, although less severe in terms of the pressure buildup $p \sim \eta^{-2 / 3}$, occurs at the expense of the magnetic energy contained in the sheet (Craig et al. 1997) and represents an equipartition between the magnetic and dynamic pressures accumulated in the reconnection region (Litvinenko \& Craig 1998). In the context of the present study, it seems likely that different forms for the background field $K$-or even differing aspect ratios in the convection cells-could produce different scalings for the dissipation rate. These possibilities, along with the longtime behavior of post saturation solutions, are currently being investigated.
P. G. W. acknowledges the financial support of a Marsden Fund Postdoctoral Fellowship. I. J. D. C. would like to thank Terry Forbes for several useful discussions and to acknowledge the support of NASA grant NAG5-1479. Thanks are given also to Jacob Heerikhuisen and Stephen Joe for their help in animating our reconnection solutions and making them available on the Web. ${ }^{2}$

\footnotetext{
${ }^{2}$ The animations can be found at http://www.math.waikato.ac.nz/ Anim/index.html.
}

Biskamp, D. 1994, Phys. Rep., 237, 181

Biskamp, D., \& Welter, H. 1980, Phys. Rev. Lett., 44, 1069

Clarke, A. 1964, Phys. Fluids, 7, 1299

Craig, I. J. D., \& Fabling, R. B. 1996, ApJ, 462, 969 1998, Phys. Plasmas, 5, 635

Craig, I. J. D., Fabling, R. B., \& Watson, P. G. 1997, ApJ, 485, 383

Craig, I. J. D., \& Henton, S. M. 1995, ApJ, 450, 280

Craig, I. J. D., Henton, S. M., \& Rickard, G. J. 1993, A\&A, 267, L39

Craig, I. J. D., \& McClymont, A. N. 1997, ApJ, 481, 996

Fabling, R. B., \& Craig, I. J. D. 1996, Phys. Plasmas, 3, 2243

Forbes, T. G., \& Priest, E. R. 1987, Rev. Geophys., 25, 1587

Inverarity, G. W., \& Priest, E. R. 1996, Phys. Plasmas, 3, 3591

Litvinenko, Y. E., \& Craig, I. J. D. 1998, ApJ, submitted

\section{REFERENCES}

McClymont, A. N., \& Craig, I. J. D. 1996, ApJ, 466, 487

Parker, E. N. 1979, Cosmical Magnetic Fields (Oxford: Clarendon Press)

Rickard, G. J., \& Craig, I. J. D. 1993, Phys. Fluids B, 5, 956

Sonnerup, B., \& Priest, E. R. 1975, J. Plasma Phys., 14, 283

Spitzer, L. 1967, The Physics of Fully Ionized Gases (2nd ed.; New York: Interscience)

Watson, P. G., \& Craig, I. J. D. 1997a, Phys. Plasmas, 4, 101 1997b, Phys. Plasmas, 4, 110 1998, ApJ, 505, 363

Watson, P. G., Priest, E. R., \& Craig, I. J. D. 1998, Geophys. Astrophys. Fluid Dyn., 88, 165

Zweibel, E. G. 1998, Phys. Plasmas, 5, 247 